hep-th/0510164

BHU-SNB/Preprint

\title{
Superfield approach to nilpotent symmetries for QED from a single restriction: an alternative to the horizontality condition
}

\author{
R.P.Malik \\ S. N. Bose National Centre for Basic Sciences, \\ Block-JD, Sector-III, Salt Lake, Calcutta-700 098, India \\ and \\ Centre of Advanced Studies, Physics Department, \\ Banaras Hindu University, Varanasi- 221 005, India \\ E-mail address: malik@bhu.ac.in
}

\begin{abstract}
We derive together the exact local, covariant, continuous and off-shell nilpotent Becchi-Rouet-Stora-Tyutin (BRST) and anti-BRST symmetry transformations for the $U(1)$ gauge field $\left(A_{\mu}\right)$, the (anti-)ghost fields $((\bar{C}) C)$ and the Dirac fields $(\psi, \bar{\psi})$ of the Lagrangian density of a four $(3+1)$-dimensional QED by exploiting a single restriction on the six $(4,2)$ dimensional supermanifold. A set of four even spacetime coordinates $x^{\mu}(\mu=0,1,2,3)$ and two odd Grassmannian variables $\theta$ and $\bar{\theta}$ parametrize this six dimensional supermanifold. The new gauge invariant restriction on the above supermanifold owes its origin to the (super) covariant derivatives and their intimate relations with the (super) 2-form curvatures $\left(\tilde{F}^{(2)}\right) F^{(2)}$ constructed with the help of 1 -form (super) gauge connections $\left(\tilde{A}^{(1)}\right) A^{(1)}$ and (super) exterior derivatives $(\tilde{d}) d$. The results obtained by exploiting (i) the horizontality condition, and (ii) one of its consistent extensions, are shown to be a simple consequence of this new single restriction on the above supermanifold. Thus, our present endeavour provides an alternative to (and, in some sense, generalization of) the horizontality condition of the usual superfield formalism applied to the derivation of BRST symmetries.
\end{abstract}

PACS numbers: 11.15.-q; 12.20.-m; 03.70.+k

Keywords: Augmented superfield formalism; gauge invariant restriction; QED with Dirac fields in 4D; off-shell nilpotent (anti-)BRST symmetries; geometrical interpretations 


\section{Introduction}

The usual superfield approach [1-6] to Becchi-Rouet-Stora-Tyutin (BRST) formalism provides the geometrical origin and interpretations for the nilpotent (anti-)BRST symmetry transformations (and their corresponding generators) for the $p$-form $(p=1,2, \ldots)$ gauge fields and corresponding (anti-)ghost fields of the $p$-form interacting gauge theories * which include matter fields as well. This approach, however, does not shed any light on the nilpotent symmetry transformations associated with the matter fields, present in the above interacting gauge theories. It has been a challenging problem to derive them cogently within the framework of the superfield approach to BRST formalism.

The above usual superfield formalism has been exploited extensively for the derivation of the nilpotent (anti-)BRST symmetries in the context of four $(3+1)$-dimensional (4D) 1-form and 2-form (non-)Abelian gauge theories which are, in general, considered on the six $(4,2)$-dimensional (6D) supermanifold [1-6]. The latter is parametrized by the superspace variables $Z^{M}=\left(x^{\mu}, \theta, \bar{\theta}\right)$ where the four even (bosonic) variables $x^{\mu}(\mu=0,1,2,3)$ correspond to the $4 \mathrm{D}$ spacetime variables and two odd Grassmannian $\left(\theta^{2}=\bar{\theta}^{2}=0, \theta \bar{\theta}+\bar{\theta} \theta=0\right)$ variables are the additional coordinates on the supermanifold. The nilpotent (anti-)BRST symmetries for the 1-form 4D non-Abelian gauge fields and the corresponding (anti-)ghost fields emerge from the horizontality condition [1-6] on the 6D supermanifold which enforces the equality $\left(\tilde{F}^{(2)}=F^{(2)}\right)$ of the 2 -form super curvature $\tilde{F}^{(2)}=\tilde{d} \tilde{A}^{(1)}+\tilde{A}^{(1)} \wedge \tilde{A}^{(1)}$ (constructed with the help of the super exterior derivative $\tilde{d}$ and the 1-form super connection $\tilde{A}^{(1)}$ ) to the ordinary 2-form curvature $F^{(2)}=d A^{(1)}+A^{(1)} \wedge A^{(1)}$ (constructed with the help of the ordinary exterior derivative $d$ and the 1-form connection $\left.A^{(1)}\right)$. The above arguments (with the theoretical arsenal of the horizontality condition) have also been applied to the case of 2-form Abelian gauge theory in a straightforward manner (see, e.g., [6] for details).

For the discussion of any arbitrary four dimensional $p$-form $\left[A^{(p)}=\frac{1}{p !}\left(d x^{\mu_{1}} \wedge d x^{\mu_{2}} \ldots \wedge \wedge\right.\right.$ $\left.\left.d x^{\mu_{p}}\right) A_{\mu_{1} \mu_{2} \ldots . . \mu_{p}}\right]$ Abelian gauge theory, within the framework of the usual superfield approach to BRST symmetries, one constructs a $(p+1)$-form super curvature $\tilde{F}^{(p+1)}=\tilde{d} \tilde{A}^{(p)}$ with the help of a super exterior derivative $\tilde{d}$ and the super $6 \mathrm{D} p$-form connection $\tilde{A}^{(p)}$ on the $6 \mathrm{D}$ supermanifold. This is subsequently equated, due to the so-called horizontality condition [1-6], to the ordinary four dimensional $(p+1)$ form curvature $F^{(p+1)}=d A^{(p)}$ constructed with the help of the ordinary exterior derivative $d=d x^{\mu} \partial_{\mu}$ (with $d^{2}=0$ ) and the ordinary $4 \mathrm{D} p$-form connection $A^{(p)}$. The covariant reduction of the $6 \mathrm{D}$ super curvature to the ordinary 4D curvature, through the equality $\tilde{F}^{(p+1)}=F^{(p+1)}$ due to the horizontality condition, leads to the derivation of the nilpotent (anti-)BRST symmetry transformations

${ }^{*}$ Such a class of 1-form gauge theories (that provide the theoretical basis for the three out of four fundamental interactions of nature) is endowed with the first class constraints in the language of Dirac's prescription for classification scheme $[7,8]$. These constraints generate the local gauge symmetries which dictate the interaction term in the theory. In fact, the interaction term arises due to the coupling of the 1-form gauge fields with the conserved Noether currents constructed by the matter (and other relevant) fields when one demands the local gauge invariance in the theory. 
for the $p$-form Abelian gauge field and the corresponding (anti)commuting (anti-)ghost fields of the given $p$-form 4D Abelian gauge theory.

The horizontality condition of the above superfield approach has been christened as the soul-flatness condition in [9] which amounts to setting equal to zero the Grassmannian components of the (anti)symmetric super curvature tensor that constitutes the super 2form $\tilde{F}^{(2)}$ (corresponding to a given 1-form gauge theory). The covariant reduction of $\tilde{F}^{(2)}$ (defined on the $6 \mathrm{D}$ supermanifold) to the ordinary 2-form curvature $F^{(2)}$ (defined on the $4 \mathrm{D}$ ordinary spacetime manifold) leads to the geometrical origin and interpretations for (i) the internal nilpotent (anti-)BRST symmetry transformations for the 4D ordinary fields as the translations of the corresponding 6D superfields along the Grassmannian directions of the 6D supermanifold, (ii) the nilpotent (anti-)BRST charges as the translation generators along $\theta$ and $\bar{\theta}$ directions of the 6D supermanifold, (iii) the nilpotency property as a couple of successive translations along a particular Grassmannian direction of the supermanifold, and (iv) the anticommutativity property of the (anti-)BRST symmetries (and their generators) as the anticommutativity encoded in the translational generators along the $\theta$ and $\bar{\theta}$ directions of the supermanifold (cf. (4.24) below). These beautiful connections between the geometrical objects on the 6D supermanifold and some key properties associated with the internal nilpotent symmetry transformations of the BRST formalism in the ordinary 4D spacetime are, however, confined only to the gauge fields and the (anti-)ghost fields of the theory within the framework of the usual superfield approach to BRST formalism [1-6].

In a very recent set of papers [10-14], the usual superfield formalism has been generalized to the augmented superfield formalism ${ }^{\dagger}$ where additional restrictions on the 6D supermanifolds have been invoked which have been found to be consistent with (and complementary to) the horizontality condition. This augmented version of the superfield approach enables one to derive the nilpotent (anti-)BRST symmetry transformations for all the fields of the (non-)Abelian gauge theories [10-14] (as well as the reparametrization invariant (supersymmetric) theories [11]) while keeping the geometrical interpretations of the (anti-)BRST symmetries (and their generators) intact. These additional restrictions on the 6D supermanifold owe their origin to the equality of (i) the conserved and gauge invariant matter currents [10] (and other conserved quantities [11]), and (ii) the gauge (i.e. BRST) invariant quantities constructed with the help of the (super) covariant derivatives [12-14]. The former restrictions allow a logically consistent derivation of the (anti-)BRST symmetry transformations for the matter fields whereas the latter lead to the derivations that are mathematically unique. Both the above extensions have their own merits and advantages.

The purpose of the present paper is to derive the off-shell nilpotent and anticommuting (anti-)BRST symmetry transformations for all the fields of the 4D QED (that includes Dirac fields as matter fields) from a single gauge (i.e. BRST) invariant restriction on the $6 \mathrm{D}$ supermanifold. We obtain all the nilpotent symmetry transformations that are derived by exploiting (i) the horizontality condition, and (ii) one of its consistent generalizations

\footnotetext{
${ }^{\dagger}$ Any mathematically consistent generalization of the usual superfield approach to BRST formalism.
} 
[10], separately. In fact, the consequences of both the above independent restrictions (i.e. (i) and (ii)) emerge very naturally from our present single restriction (cf. (4.1) below). Our present investigation is essential primarily on four counts. First and foremost, the horizontality condition, as discussed earlier, does not shed any light on the derivation of the nilpotent symmetry transformations associated with the matter fields of a given interacting gauge theory whereas our present single restriction on the 6D supermanifold does precisely that. Second, the single restriction (cf. (4.1) below) imposed on the 6D supermanifold is a gauge (i.e. BRST) invariant condition that is more physical than the horizontality condition which happens to be intrinsically a gauge covariant restriction. Third, our present single restriction is a nice simplification of our previous attempts [10-14] where two separate restrictions were imposed on the supermanifold for the derivation of all the nilpotent transformations in the context of (non-)Abelian gauge (and reparametrization invariant) theories. Finally, the horizontality condition and one of its consistent extensions [10] are, in some sense, unified together in our present single restriction. Thus, the imposition of our present single restriction (cf. (4.1) below) on the 6D supermanifold is aesthetically and physically more appealing than the imposition of the horizontality condition alone.

The contents of our present paper are organized as follows. In section 2, we set up the notations and conventions by recapitulating the bare essentials of the (anti-)BRST symmetry transformations in the framework of Lagrangian formulation for QED with Dirac fields. Section 3 is devoted to the definition of suitable superfields and their expansions, in terms of the basic and some secondary fields, along the Grassmannian directions of the supermanifold. The central results of our investigation are contained in section 4 where we derive the nilpotent (anti-)BRST transformations for all the fields of the above QED from a single restriction (cf. (4.1) below) on the supermanifold. Finally, we make some concluding remarks and point out a few future directions for further investigations in section 5 .

\section{2 (Anti-)BRST symmetries in Lagrangian formulation: a brief sketch}

Let us begin with the (anti-)BRST invariant Lagrangian density $\mathcal{L}_{B}$ for the interacting four $(3+1)$-dimensional $(4 \mathrm{D}) U(1)$ gauge theory $(\mathrm{QED})$ in the Feynman gauge ${ }^{\ddagger}[9,15,16]$

$$
\mathcal{L}_{B}=-\frac{1}{4} F^{\mu \nu} F_{\mu \nu}+\bar{\psi}\left(i \gamma^{\mu} D_{\mu}-m\right) \psi+B(\partial \cdot A)+\frac{1}{2} B^{2}-i \partial_{\mu} \bar{C} \partial^{\mu} C
$$

where $D_{\mu} \psi=\partial_{\mu} \psi+i e A_{\mu} \psi$ is the covariant derivative on the Dirac field $\psi(x)$ with charge $e$ and mass $m$. The $U(1)$ gauge field $A_{\mu}$ couples to the matter conserved current $J_{\mu}=$ $\bar{\psi} \gamma_{\mu} \psi$ (constructed by the Dirac fields $(\psi, \bar{\psi})$ ) with the coupling strength $e$. This coupling

\footnotetext{
${ }^{\ddagger}$ We adopt here the notations and conventions such that the flat Monkowskian metric $\eta_{\mu \nu}=$ diag $(+1,-1,-1,-1)$ for the $4 \mathrm{D}$ spacetime manifold and $F_{0 i}=\partial_{0} A_{i}-\partial_{i} A_{0}=E_{i}, F_{i j}=\epsilon_{i j k} B_{k}, B_{i}=\frac{1}{2} \epsilon_{i j k} F_{j k}$ are the electric $\left(E_{i}\right)$ and magnetic $\left(B_{i}\right)$ components of the field strength tensor $F_{\mu \nu}$. Here $\epsilon_{i j k}$ is the totally antisymmetric Levi-Civita tensor (with $\epsilon_{123}=+1$ ) on the 3D subspace of the 4D Minkowskian space. Furthermore, the Greek indices $\mu, \nu, \ldots=0,1,2,3$, present in (2.1), stand for the spacetime directions and Latin indices $i, j, k \ldots=1,2,3$ correspond only to the space directions on the $4 \mathrm{D}$ spacetime manifold.
} 
generates an interaction term $-e \bar{\psi} \gamma^{\mu} A_{\mu} \psi$ in the theory which exists basically due to the requirement of the local $U(1)$ gauge invariance. The $\gamma$ 's in (2.1) are the usual Dirac $4 \times 4$ matrices. The 2-form $F^{(2)}=d A^{(1)}=\frac{1}{2}\left(d x^{\mu} \wedge d x^{\nu}\right) F_{\mu \nu}$, constructed with the help of the exterior derivative $d=d x^{\mu} \partial_{\mu}$ (with $d^{2}=0$ ) and 1-form $A^{(1)}=d x^{\mu} A_{\mu}$, defines the field strength tensor $F_{\mu \nu}=\partial_{\mu} A_{\nu}-\partial_{\nu} A_{\mu}$ for the $U(1)$ gauge field $A_{\mu}$. The Nakanishi-Lautrup auxiliary field $B$ linearizes the gauge-fixing term $-\frac{1}{2}(\partial \cdot A)^{2}$ of the Lagrangian density (2.1) and the fermionic (i.e. $C^{2}=\bar{C}^{2}=0, C \bar{C}+\bar{C} C=0$ ) (anti-)ghost fields $(\bar{C}) C$ are required to maintain the unitarity and "quantum" gauge (i.e. BRST) invariance together, for a given physical process, at any arbitrary order of perturbative computation ${ }^{\S}$.

The above Lagrangian density (2.1) for QED with Dirac fields, respects the following infinitesimal, off-shell nilpotent $\left(s_{(a) b}^{2}=0\right)$, anticommuting $\left(s_{b} s_{a b}+s_{a b} s_{b}=0\right)$, local,

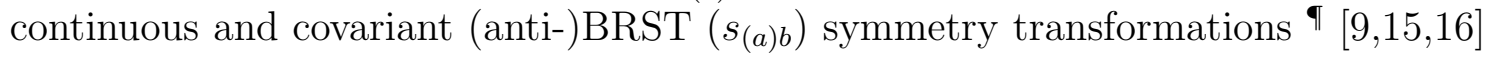

$$
\begin{array}{llrrr}
s_{b} A_{\mu} & =\partial_{\mu} C & s_{b} C=0 & s_{b} \bar{C}=i B & s_{b} \psi=-i e C \psi \\
s_{b} \bar{\psi} & =-i e \bar{\psi} C & s_{b} B=0 & s_{b} F_{\mu \nu}=0 & s_{b}(\partial \cdot A)=\square C \\
s_{a b} A_{\mu}=\partial_{\mu} \bar{C} & s_{a b} \bar{C}=0 & s_{a b} C=-i B & s_{a b} \psi=-i e \bar{C} \psi \\
s_{a b} \bar{\psi}=-i e \bar{\psi} \bar{C} & s_{a b} B=0 & s_{a b} F_{\mu \nu}=0 & s_{a b}(\partial \cdot A)=\square \bar{C}
\end{array}
$$

because it transforms to a total derivative. Some noteworthy points, at this juncture, are in order now. First, under the nilpotent (anti-)BRST transformations, the kinetic energy term of the (non-)Abelian gauge fields remains invariant. More precisely, for the Abelian gauge theory, it is the field strength tensor $F_{\mu \nu}$ itself that remains unchanged. Second, the gaugefixing term $(\partial \cdot A)$, on the other hand, transforms under the (anti-)BRST transformations. Finally, the cohomological operator $d=d x^{\mu} \partial_{\mu}$ (with $d^{2}=0$ ) and the nilpotent $\left(s_{(a) b}^{2}=0\right)$ (anti-)BRST transformations $s_{(a) b}$ are inter-connected. This is due to the fact that the electric $E_{i}$ and magnetic $B_{i}$ fields (which are components of $F_{\mu \nu}$ ) remain invariant under the transformations $s_{(a) b}$ and they owe their origin, primarily, to the nilpotent $\left(d^{2}=0\right)$ cohomological operator $d$ because $F^{(2)}=d A^{(1)}=\frac{1}{2}\left(d x^{\mu} \wedge d x^{\nu}\right) F_{\mu \nu}$.

According to the Noether's theorem, the above continuous symmetry transformations lead to the derivation of the conserved (anti-)BRST charges $Q_{(a) b}$ which are found to be nilpotent $\left(Q_{(a) b}^{2}=0\right)$ of order two. These charges generate the above continuous nilpotent transformations. For a generic local field $\Omega=A_{\mu}, C, \bar{C}, \psi, \bar{\psi}, B$ of the Lagrangian density (2.1), the infinitesimal transformations (2.2) can be expressed in terms of $Q_{(a) b}$, as

$$
s_{r} \Omega(x)=-i\left[\Omega(x), Q_{r}\right]_{ \pm} \quad r=b, a b
$$

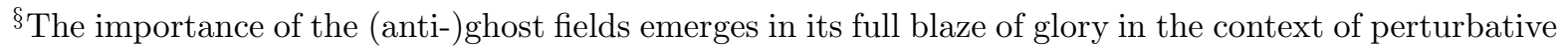
computations, connected with a given physical process, that is allowed by the interacting non-Abelian gauge theory. In fact, for the proof of unitarity of such a kind of physical process, one requires a Feynman loop diagram constructed by purely the fermionic (anti-)ghost fields corresponding to each such loop diagram existing in the theory due to a purely bosonic non-Abelian gauge (gluon) field (see, e.g., [17] for details).

"We follow here the notations adopted in $[15,16]$. In fact, the BRST prescription is to replace the local gauge parameter of the original gauge theory by an anticommuting $(\eta C+C \eta=0, \eta \psi+\psi \eta=0$, etc. $)$ spacetime independent parameter $\eta$ and the (anti-)ghost fields. Thus, in its totality, the (anti-)BRST transformations $\delta_{(A) B}$ are a product $\left(\delta_{(A) B}=\eta s_{(a) b}\right)$ of $\eta$ and the nilpotent $s_{(a) b}^{2}=0$ transformations $s_{(a) b}$. 
where the subscripts $(+)-$, on the square bracket, stand for this bracket to correspond to an (anti)commutator for the generic local field $\Omega(x)$ of the Lagrangian density (2.1) being (fermionic)bosonic in nature. For our present discussions, the exact expressions for the conserved, nilpotent and anticommuting $\left(Q_{b} Q_{a b}+Q_{a b} Q_{b}=0\right)\left(\right.$ anti-)BRST charges $Q_{(a) b}$ are not important but their explicit forms can be found in $[9,15,16]$.

\section{From ordinary basic fields to superfields: super expansion}

To derive the above local, continuous, covariant, nilpotent and anticommuting (anti-)BRST infinitesimal transformations (2.2) within the framework of superfield formalism, first of all, we generalize the basic local fields $A_{\mu}, C, \bar{C}, \psi, \bar{\psi}$ of the Lagrangian density (2.1), defined on the $4 \mathrm{D}$ spacetime manifold, to the corresponding superfields $\mathcal{B}_{\mu}, \mathcal{F}, \overline{\mathcal{F}}, \Psi, \bar{\Psi}$ defined on the six dimensional supermanifold parametrized by the superspace variables $Z^{M}=\left(x^{\mu}, \theta, \bar{\theta}\right)$. These superfields can be expanded in terms of the basic fields $\left(A_{\mu}, C, \bar{C}, \psi, \bar{\psi}\right)$ and extra secondary fields, along the Grassmannian directions, as follows $[4,3,10]$

$$
\begin{aligned}
\mathcal{B}_{\mu}(x, \theta, \bar{\theta}) & =A_{\mu}(x)+\theta \bar{R}_{\mu}(x)+\bar{\theta} R_{\mu}(x)+i \theta \bar{\theta} S_{\mu}(x) \\
\mathcal{F}(x, \theta, \bar{\theta}) & =C(x)+i \theta \bar{B}_{1}(x)+i \bar{\theta} B_{2}(x)+i \theta \bar{\theta} s(x) \\
\overline{\mathcal{F}}(x, \theta, \bar{\theta}) & =\bar{C}(x)+i \theta \bar{B}_{2}(x)+i \bar{\theta} B_{1}(x)+i \theta \bar{\theta} \bar{s}(x) \\
\Psi(x, \theta, \bar{\theta}) & =\psi(x)+i \theta \bar{b}_{1}(x)+i \bar{\theta} b_{2}(x)+i \theta \bar{\theta} f(x) \\
\bar{\Psi}(x, \theta, \bar{\theta}) & =\bar{\psi}(x)+i \theta \bar{b}_{2}(x)+i \bar{\theta} b_{1}(x)+i \theta \bar{\theta} \bar{f}(x) .
\end{aligned}
$$

It is obvious that the superfield $\mathcal{B}_{\mu}(x, \theta, \bar{\theta})$ is bosonic and the rest of the above superfields are fermionic (i.e. $\mathcal{F}^{2}=\overline{\mathcal{F}}^{2}=\Psi^{2}=\bar{\Psi}^{2}=0$ ) in nature.

A few salient features of the above expansions are as follows:

(i) In the limit $(\theta, \bar{\theta}) \rightarrow 0$, we do retrieve the basic fields of the Lagrangian density (2.1) that are defined on the $4 \mathrm{D}$ ordinary Minkowskian spacetime manifold.

(ii) In the above expansion, the total number of fermionic fields $\left(\psi, \bar{\psi}, f, \bar{f}, C, \bar{C}, s, \bar{s}, R_{\mu}, \bar{R}_{\mu}\right)$ do match with the bosonic fields $\left(b_{1}, \bar{b}_{1}, b_{2}, \bar{b}_{2}, B_{1}, \bar{B}_{1}, B_{2}, \bar{B}_{2}, A_{\mu}, S_{\mu}\right)$.

(iii) The above straightforward observation in (ii) is an essential requirement for any arbitrary superfield theory to be discussed in the framework of supersymmetry.

It is important to generalize the exterior derivative $d=d x^{\mu} \partial_{\mu}$ and the 1 -form connection $A^{(1)}=d x^{\mu} A_{\mu}$, defined on the ordinary 4D Minkowskian flat manifold, to the six $(4,2)-$ dimensional supermanifold. This is required because, as discussed and emphasized earlier after (2.2), the above geometrical quantities have relevance with the (anti-)BRST symmetry transformations. Thus, these quantities on the above supermanifold, are

$$
\begin{aligned}
& \tilde{d}=d Z^{M} \partial_{M}=d x^{\mu} \partial_{\mu}+d \theta \partial_{\theta}+d \bar{\theta} \partial_{\bar{\theta}} \\
& \tilde{A}^{(1)}=d Z^{M} \tilde{A}_{M}=d x^{\mu} \mathcal{B}_{\mu}(x, \theta, \bar{\theta})+d \theta \overline{\mathcal{F}}(x, \theta, \bar{\theta})+d \bar{\theta} \mathcal{F}(x, \theta, \bar{\theta})
\end{aligned}
$$

which reduce to $d=d x^{\mu} \partial_{\mu}$ and $A^{(1)}=d x^{\mu} A_{\mu}$ in the limit $(\theta, \bar{\theta}) \rightarrow 0$. It is clear, therefore, that (i) $\tilde{d}$ and $\tilde{A}^{(1)}$ are a set of consistent $6 \mathrm{D}$ superspace generalization of the $4 \mathrm{D}$ quantities $d$ and $A^{(1)}$ defined on the ordinary space, and (ii) the superspace derivative $\partial_{M}$ 
and supervector superfield $\tilde{A}_{M}$ have the component multiplets $\left(\partial_{\mu}, \partial_{\theta}, \partial_{\bar{\theta}}\right)$ and $\left(\mathcal{B}_{\mu}, \mathcal{F}, \overline{\mathcal{F}}\right)$, respectively. Both the quantities, defined in (3.2), would be useful in the next section.

\section{Gauge invariant restriction on supermanifold: nilpotent symmetries}

To provide the geometrical interpretation for the nilpotent symmetry transformations $(2.2)$ within the framework of the superfield approach to BRST formalism, we have to exploit a certain specific restriction on the supermanifold. To this end in mind, we begin with the following gauge invariant restriction on the six (4,2)-dimensional supermanifold:

$$
\bar{\Psi}(x, \theta, \bar{\theta}) \tilde{D} \tilde{D} \Psi(x, \theta, \bar{\theta})=\bar{\psi}(x) D D \psi(x)
$$

where the (super) covariant derivatives on the six-dimensional supermanifold (i.e. $\tilde{D}$ ) and ordinary 4D Minkowskian spacetime manifold (i.e. D), are

$$
\tilde{D}=\tilde{d}+i \text { e } \tilde{A}^{(1)}(x, \theta, \bar{\theta}) \quad D=d+i \text { e } A^{(1)}(x) .
$$

In the above, the symbols $\tilde{d}$ and $\tilde{A}^{(1)}$ are defined in (3.2) on the (4,2)-dimensional supermanifold and corresponding 4D quantities are: $d=d x^{\mu} \partial_{\mu}, A^{(1)}=d x^{\mu} A_{\mu}$.

It is obvious that the r.h.s. of $(4.1)$ is a $U(1)$ gauge invariant quantity which can be explicitly expressed, in terms of the 2-form curvature $F^{(2)}=d A^{(1)}$, as

$$
\bar{\psi}(x) D D \psi(x)=\frac{1}{2} i e\left(d x^{\mu} \wedge d x^{\nu}\right) \bar{\psi}(x) F_{\mu \nu}(x) \psi(x) \equiv i e \bar{\psi} F^{(2)} \psi .
$$

It will be noted that (i) the r.h.s. of (4.3) possesses only the 2 -form differentials $\frac{1}{2}\left(d x^{\mu} \wedge d x^{\nu}\right)$ in terms of spacetime variables, and (ii) the well-known relation $D D \psi=i e F^{(2)} \psi$ has been used in the above derivation. In contrast, the 1.h.s will lead to the 2-form differentials $\frac{1}{2}\left(d Z^{M} \wedge d Z^{N}\right)$ which will contain all the possible combinations of 2-forms, constructed by superspace differentials (i.e. $d x^{\mu} \wedge d x^{\nu}, d x^{\mu} \wedge d \theta, d x^{\mu} \wedge d \bar{\theta}, d \bar{\theta} \wedge d \bar{\theta}, d \theta \wedge d \bar{\theta}, d \theta \wedge d \theta$ ).

The explicit form of the l.h.s., in terms of the component multiplet superfields $\mathcal{B}_{\mu}, \mathcal{F}, \overline{\mathcal{F}}$, the superspace differentials and the partial derivatives $\partial_{\mu}, \partial_{\theta}, \partial_{\bar{\theta}}$, can be written as

$$
\begin{aligned}
& \bar{\Psi}\left(d x^{\mu}\right)\left(\partial_{\mu}+i e \mathcal{B}_{\mu}\right) \wedge\left[d x^{\nu}\left(\partial_{\nu}+i e \mathcal{B}_{\nu}\right) \Psi+d \theta\left(\partial_{\theta}+i e \overline{\mathcal{F}}\right) \Psi+d \bar{\theta}\left(\partial_{\bar{\theta}}+i e \mathcal{F}\right) \Psi\right] \\
&+ \bar{\Psi}(d \theta)\left(\partial_{\theta}+i e \overline{\mathcal{F}}\right) \wedge\left[d x^{\mu}\left(\partial_{\mu}+i e \mathcal{B}_{\mu}\right) \Psi+d \theta\left(\partial_{\theta}+i e \overline{\mathcal{F}}\right) \Psi+d \bar{\theta}\left(\partial_{\bar{\theta}}+i e \mathcal{F}\right) \Psi\right] \\
&+\bar{\Psi}(d \bar{\theta})\left(\partial_{\bar{\theta}}+i e \mathcal{F}\right) \wedge\left[d x^{\mu}\left(\partial_{\mu}+i e \mathcal{B}_{\mu}\right) \Psi+d \theta\left(\partial_{\theta}+i e \overline{\mathcal{F}}\right) \Psi+d \bar{\theta}\left(\partial_{\bar{\theta}}+i e \mathcal{F}\right) \Psi\right] .
\end{aligned}
$$

The expansion of the above equation would lead to the coefficients of $\left(d Z^{M} \wedge d Z^{N}\right)$ where the superspace variable $Z^{M}=\left(x^{\mu}, \theta, \bar{\theta}\right)$. It is straightforward to note that the 2-form, constructed only with the spacetime differentials $\left(d x^{\mu} \wedge d x^{\nu}\right)$, would match with the similar kind of 2-form emerging from the r.h.s. (cf. (4.3)). The rest of the components of the super 2-form (with the Grassmannian differentials) will be set equal to zero due to (4.1).

For algebraic convenience, it is useful to first collect the coefficients of $(d \theta \wedge d \theta)$ which can be succinctly expressed as follows:

$$
-i e(d \theta \wedge d \theta)\left[i \bar{\psi} \bar{B}_{2} \psi+\theta\left(L_{1}\right)+\bar{\theta}\left(M_{1}\right)-\theta \bar{\theta}\left(N_{1}\right)\right]
$$


where the explicit expressions for $L_{1}, M_{1}$ and $N_{1}$ are

$$
\begin{aligned}
& L_{1}=\bar{\psi} \bar{B}_{2} \bar{b}_{1}-\bar{b}_{2} \bar{B}_{2} \psi \quad M_{1}=\bar{\psi} \bar{B}_{2} b_{2}-b_{1} \bar{B}_{2} \psi-i \bar{\psi} \bar{s} \psi \\
& N_{1}=\bar{\psi} \bar{B}_{2} f+\bar{\psi} \bar{s}_{1}+\bar{f} \bar{B}_{2} \psi+\bar{b}_{2} \bar{s} \psi+i \bar{b}_{2} \bar{B}_{2} b_{2}-i b_{1} \bar{B}_{2} \bar{b}_{1} \text {. }
\end{aligned}
$$

In the above computation, we have exploited the following inputs:

$$
\partial_{\theta} \partial_{\bar{\theta}} \Psi=-i f \quad \partial_{\theta} \Psi=i \bar{b}_{1}+i \bar{\theta} f \quad \partial_{\bar{\theta}} \Psi=i b_{2}-i \theta f \quad \partial_{\theta} \mathcal{F}=i \bar{B}_{1}+i \bar{\theta} s .
$$

Ultimately, we have to set equal to zero, separately and independently, the coefficients of $(d \theta \wedge d \theta),[(d \theta \wedge d \theta)(\theta)],[(d \theta \wedge d \theta)(\bar{\theta})]$ and $[(d \theta \wedge d \theta)(\theta \bar{\theta})]$. Restrictions emerging from these conditions, for $e \neq 0, \psi \neq 0, \bar{\psi} \neq 0$, are

$$
\bar{B}_{2}=0 \quad \bar{s}=0
$$

The above values, substituted in (3.1), imply that the fermionic superfield $\overline{\mathcal{F}}(x, \theta, \bar{\theta})$ becomes an anti-chiral superfield because it is constrained to be independent of $\theta$.

In an exactly similar fashion, we can compute the coefficients of $(d \bar{\theta} \wedge d \bar{\theta})$. These are concisely expressed by the following equation

$$
+i e(d \bar{\theta} \wedge d \bar{\theta})\left[-i \bar{\psi} B_{2} \psi+\theta\left(L_{2}\right)+\bar{\theta}\left(M_{2}\right)+\theta \bar{\theta}\left(N_{2}\right)\right]
$$

where the detailed expressions for $L_{2}, M_{2}, N_{2}$, are

$$
\begin{aligned}
& L_{2}=-\bar{\psi} B_{2} \bar{b}_{1}+\bar{b}_{2} B_{2} \psi \quad M_{2}=-\bar{\psi} B_{2} b_{2}+b_{1} B_{2} \psi+i \bar{\psi} s \psi \\
& N_{2}=\bar{\psi} B_{2} f+\bar{\psi} s \bar{b}_{1}+\bar{f} B_{2} \psi+\bar{b}_{2} s \psi+i \bar{b}_{2} B_{2} b_{2}+i b_{1} B_{2} \bar{b}_{1} .
\end{aligned}
$$

To retain the restriction imposed on supermanifold in (4.1), it is straightforward to note that the coefficients of $(d \bar{\theta} \wedge d \bar{\theta}),[(d \bar{\theta} \wedge d \bar{\theta})(\theta)],[(d \bar{\theta} \wedge d \bar{\theta})(\bar{\theta})]$ and $[(d \bar{\theta} \wedge d \bar{\theta})(\theta \bar{\theta})]$ in $(4.9)$ would be equal to zero separately and independently. These finally imply (for $e \neq 0, \psi \neq 0, \bar{\psi} \neq 0$ )

$$
B_{2}=0 \quad s=0 .
$$

This entails upon the fermionic superfield $\mathcal{F}(x, \theta, \bar{\theta})$ to become chiral in nature. Results of (4.8) and (4.11) lead to the following expansions for the fermionic superfields in (3.1):

$$
\mathcal{F}^{(c)}(x, \theta)=C(x)+i \theta \bar{B}_{1}(x) \quad \overline{\mathcal{F}}^{(a c)}(x, \bar{\theta})=\bar{C}(x)+i \bar{\theta} B_{1}(x) .
$$

The above expansions will be used in our further computations.

Now we focus on the computations of the coefficients of $(d \theta \wedge d \bar{\theta})$. These are expressed in terms of the fermionic superfield expansion of (4.12), as

$$
-(d \theta \wedge d \bar{\theta})\left[\bar{\Psi}\left\{\left(\partial_{\theta}+i e \overline{\mathcal{F}}^{(a c)}\right)\left(\partial_{\bar{\theta}}+i e \mathcal{F}^{(c)}\right)+\left(\partial_{\bar{\theta}}+i e \mathcal{F}^{(c)}\right)\left(\partial_{\theta}+i e \overline{\mathcal{F}}^{(a c)}\right)\right\} \Psi\right] .
$$

The above equation can be simplified to finally yield

$$
-i e(d \theta \wedge d \bar{\theta})\left[\bar{\Psi}\left(\partial_{\theta} \mathcal{F}^{(c)}+\partial_{\bar{\theta}} \overline{\mathcal{F}}^{(a c)}\right) \Psi\right]=0
$$


where we have used: $\partial_{\theta} \partial_{\bar{\theta}}+\partial_{\bar{\theta}} \partial_{\theta}=0, C \bar{C}=-\bar{C} C, \mathcal{F}^{(c)} \overline{\mathcal{F}}^{(a c)}=-\overline{\mathcal{F}}^{(a c)} \mathcal{F}^{(c)}$. This condition can be satisfied if and only if $B_{1}(x)+\bar{B}_{1}(x)=0$. We are free to choose $B_{1}(x)=-\bar{B}_{1}(x)=$ $B(x)$ of the Lagrangian density (2.1). Thus, we have (for $\Psi \neq 0, \bar{\Psi} \neq 0, e \neq 0$ )

$$
\begin{aligned}
& \mathcal{F}^{(c)}=C(x)-i \theta B(x) \equiv C(x)+\theta\left(s_{a b} C(x)\right) \\
& \overline{\mathcal{F}}^{(a c)}=\bar{C}(x)+i \bar{\theta} B(x) \equiv \bar{C}(x)+\bar{\theta}\left(s_{b} \bar{C}(x)\right)
\end{aligned}
$$

which lead to the derivation of the (anti-)BRST transformations (2.2) for the (anti-)ghost fields $(\bar{C}) C$ in the framework of the superfield formalism with restriction (4.1).

We collect the coefficients of $\left(d x^{\mu} \wedge d \theta\right)$ and $\left(d x^{\mu} \wedge d \bar{\theta}\right)$ from (4.4) and set them equal to zero to maintain the consistency with the restriction (4.1). These imply

$$
i e\left(d x^{\mu} \wedge d \theta\right)\left[\bar{\Psi}\left(\partial_{\theta} \mathcal{B}_{\mu}-\partial_{\mu} \overline{\mathcal{F}}^{(a c)}\right) \Psi\right]=0 \quad i e\left(d x^{\mu} \wedge d \bar{\theta}\right)\left[\bar{\Psi}\left(\partial_{\bar{\theta}} \mathcal{B}_{\mu}-\partial_{\mu} \mathcal{F}^{(c)}\right) \Psi\right]=0
$$

The above requirements, using the expansions (3.1) and (4.15), lead to

$$
R_{\mu}(x)=\partial_{\mu} C(x) \quad \bar{R}_{\mu}(x)=\partial_{\mu} \bar{C}(x) \quad S_{\mu}(x)=\partial_{\mu} B(x) .
$$

Substitution of these values in (3.1) leads to the derivation of (anti-)BRST symmetry transformations (2.2) for the $U(1)$ gauge field $A_{\mu}$, as the superfield $\mathcal{B}_{\mu} \rightarrow \mathcal{B}_{\mu}^{(g)}$. That is:

$$
\mathcal{B}_{\mu}^{(g)}(x, \theta, \bar{\theta})=A_{\mu}(x)+\theta\left(s_{a b} A_{\mu}(x)\right)+\bar{\theta}\left(s_{b} A_{\mu}(x)\right)+\theta \bar{\theta}\left(s_{b} s_{a b} A_{\mu}(x)\right) .
$$

It is worthwhile to emphasize that (i) unlike the fermionic superfields $(\overline{\mathcal{F}}, \mathcal{F})$ which reduce to (anti-)chiral superfields after the application of the restriction (4.1), the bosonic superfield $\mathcal{B}_{\mu}$ retains its general form (i.e. $\mathcal{B}_{\mu} \rightarrow \mathcal{B}_{\mu}^{(g)}$ ) even after application of (4.1), and (ii) the expansions in (4.15) and (4.18) have been obtained in earlier works [4,3,10] by exploiting the horizontality condition " on the six $(4,2)$-dimensional supermanifold.

Finally, let us compute the coefficients of the 2-form differentials $\left(d x^{\mu} \wedge d x^{\nu}\right)$, constructed by the spacetime variables. The equality that emerges from l.h.s. and r.h.s., is

$$
\frac{1}{2} i e\left(d x^{\mu} \wedge d x^{\nu}\right) \bar{\Psi}\left(\partial_{\mu} B_{\nu}^{(g)}-\partial_{\nu} B_{\mu}^{(g)}\right) \Psi=\frac{1}{2} i e\left(d x^{\mu} \wedge d x^{\nu}\right) \bar{\psi}\left(\partial_{\mu} A_{\nu}-\partial_{\nu} A_{\mu}\right) \psi
$$

It is straightforward to check, with the help of $R_{\mu}=\partial_{\mu} C, \bar{R}_{\mu}=\partial_{\mu} \bar{C}, S_{\mu}=\partial_{\mu} B$, that $\partial_{\mu} B_{\nu}^{(g)}-\partial_{\nu} B_{\mu}^{(g)}=\partial_{\mu} A_{\nu}-\partial_{\nu} A_{\mu}$. Thus, the restriction, that emerges from (4.19), is **

$$
\bar{\Psi}(x, \theta, \bar{\theta}) \Psi(x, \theta, \bar{\theta})=\bar{\psi}(x) \psi(x) \Rightarrow i \theta\left(L_{3}\right)+i \bar{\theta}\left(M_{3}\right)+i \theta \bar{\theta}\left(N_{3}\right)=0
$$

\footnotetext{
"In the horizontality condition $\tilde{F}^{(2)}=F^{(2)}$, the super 2-form curvature (i.e. $\tilde{F}^{(2)}=\tilde{d} \tilde{A}^{(1)}=\frac{1}{2}\left(d Z^{M} \wedge\right.$
} $\left.d Z^{N}\right) \tilde{F}_{M N}$ ) and the ordinary 2-form curvature (i.e. $F^{(2)}=d A^{(1)}=\frac{1}{2}\left(d x^{\mu} \wedge d x^{\nu}\right) F_{\mu \nu}$ ), are equated on the supermanifold where $\tilde{d}$ and $\tilde{A}^{(1)}$ are defined in (3.2). This restriction implies $R_{\mu}=\partial_{\mu} C, \bar{R}_{\mu}=\partial_{\mu} \bar{C}, s=$ $\bar{s}=0, S_{\mu}=\partial_{\mu} B, B_{1}+\bar{B}_{1}=0, B_{2}=\bar{B}_{2}=0$ in (3.1). Thus, these values entail upon the expansions (3.1) to reduce to (4.15) and (4.18). It is obvious that the horizontality condition leads to the derivation of the nilpotent (anti-)BRST transformations $s_{(a) b}$ only for the gauge and (anti-)ghost fields of the theory.

** It is worth emphasizing that the relation (4.20) cannot emerge from the gauge covariant version (i.e. $\tilde{D} \tilde{D} \Psi(x, \theta, \bar{\theta})=D D \psi(x))$ of the gauge invariant restriction (4.1) on the 6D supermanifold. 
where the explicit forms of $L_{3}, M_{3}$ and $N_{3}$ are

$$
L_{3}=\bar{b}_{2} \psi-\bar{\psi} \bar{b}_{1} \quad M_{3}=b_{1} \psi-\bar{\psi} b_{2} \quad N_{3}=\bar{f} \psi+\bar{\psi} f+i \bar{b}_{2} b_{2}-i b_{1} \bar{b}_{1}
$$

In the above, the expansions for the fermionic superfields $(\bar{\Psi}, \Psi)$, listed in $(3.1)$, have been taken into account for computation of the l.h.s. $\Psi(x, \theta, \bar{\theta}) \Psi(x, \theta, \bar{\theta})$.

At this juncture, it is worthwhile to mention that the simple relationship quoted in (4.20) does not emerge when one attempts to derive the nilpotent (anti-)BRST symmetry transformations for all the fields of a given 1-form 4D non-Abelian gauge theory where there is an interaction between the 1-form non-Abelian gauge field and the Dirac fields. In fact, the non-Abelian nature of the theory leads to a whole range of interesting complications when one exploits the restriction (4.1) on the 6D supermanifold. However, the accurate computation, ultimately, leads to the derivation of the exact values for the $b_{1}, \bar{b}_{1}, b_{2}, \bar{b}_{2}, f$ and $\bar{f}$ present in the expansions of the superfields $\Psi(x, \theta, \bar{\theta})$ and $\bar{\Psi}(x, \theta, \bar{\theta})$ (cf. (3.1)) for the non-Abelian gauge theory [18]. This, in turn, leads to the expansions for the fermionic superfields $\Psi(x, \theta, \bar{\theta})$ and $\bar{\Psi}(x, \theta, \bar{\theta})$ in terms of the nilpotent (anti-)BRST symmetry transformations for the Dirac fields of the interacting 1-form non-Abelian gauge theory [18]. Thus, the gauge (i.e. BRST) invariant restriction (4.1) generates the nilpotent (anti-)BRST transformations for all the fields of the interacting Abelian as well as non-Abelian gauge theories where there is an explicit coupling between the matter fields and gauge fields.

It is clear that $L_{3}, M_{3}$ and $N_{3}$ of (4.21) should be separately and independently set equal to zero to maintain the sanctity of equation (4.1) on the $6 \mathrm{D}$ supermanifold. One of the possible solutions to the conditions: $L_{3}=0, M_{3}=0$ and $N_{3}=0$, is [10]

$$
\begin{aligned}
& b_{1}=-e \bar{\psi} C \quad b_{2}=-e C \psi \quad \bar{b}_{1}=-e \bar{C} \psi \quad \bar{b}_{2}=-e \bar{\psi} \bar{C} \\
& f=-i e[B+e \bar{C} C] \psi \quad \bar{f}=+i e \bar{\psi}[B+e C \bar{C}] \text {. }
\end{aligned}
$$

The solutions, listed in (4.22), form a set of consistent solutions and, these values, in some sense, are very logical ${ }^{\dagger \dagger}$. To elaborate on the above solutions to be a logical one, let us first focus on $L_{3}=0$ which implies $\bar{b}_{2} \psi=\bar{\psi} \bar{b}_{1}$. A smart and judicious guess will be to choose the bosonic components $\bar{b}_{2}$ and $\bar{b}_{1}$ (of the expansion in (3.1)) to be proportional to the fermionic fields $\bar{\psi}$ and $\psi$, respectively. The latter fields can be made to be bosonic in nature only by bringing in the fermionic $\left(C^{2}=\bar{C}^{2}=0\right)$ (anti-)ghost fields $(\bar{C}) C$ of the theory. There is no other possible choice because the other fermionic fields $\left(\psi^{2}=0, \bar{\psi}^{2}=0\right)$ of the theory can not do the job. In exactly similar fashion, all the other choices in (4.22) have been made with an appropriate factors of the constants $i$ and $e$ thrown in.

It is worthwhile to lay stress, at this stage, that in our earlier works $[10,11]$ on the consistent extension of the usual superfield approach to BRST formalism (endowed with

${ }^{\dagger \dagger}$ To be precise, the solutions in (4.22) are not the unique set of solutions. This is due to the fact that the signs and appropriate factors of $i$ and $e$ are not determined mathematically in a unique fashion. To obtain the unique set of solutions, the gauge invariant constraint on the six dimensional supermanifold is: $\bar{\Psi}(x, \theta, \bar{\theta})\left(\tilde{d}+i e \tilde{A}_{(h)}^{(1)}\right) \Psi(x, \theta, \bar{\theta})=\bar{\psi}(x)\left(d+i e A^{(1)}\right) \psi(x)$ where $\tilde{A}_{(h)}^{(1)}=d x^{\mu} \mathcal{B}_{\mu}^{(g)}+d \theta \overline{\mathcal{F}}^{(a c)}+d \bar{\theta} \mathcal{F}^{(c)}$. This restriction on $6 \mathrm{D}$ supermanifold has been exploited in our recent work (see, e.g., [12,13] for details). 
the horizontality condition alone [1-6]), we exploited an additional new restriction on the $6 \mathrm{D}$ supermanifold by requiring the super matter current $\tilde{J}_{\mu}=\bar{\Psi}(x, \theta, \bar{\theta}) \gamma_{\mu} \Psi(x, \theta, \bar{\theta})$ to be equal to the $U(1)$ gauge invariant and conserved matter current $J_{\mu}=\bar{\psi}(x) \gamma_{\mu} \psi(x)$. This led exactly to the same kind of conditions on the component fields of the expansion of $\Psi$ and $\bar{\Psi}$, as captured in $L_{3}=M_{3}=N_{3}=0$. This happened because of the fact that both the quantities, $\bar{\psi} \gamma_{\mu} \psi$ and $\bar{\psi} \psi$, are $U(1)$ gauge (and, therefore, BRST) invariant quantities. The most interesting feature of our present investigation is the crucial fact that the condition $\bar{\Psi}(x, \theta, \bar{\theta}) \Psi(x, \theta, \bar{\theta})=\bar{\psi}(x) \psi(x)$ comes out automatically from the single restriction (4.1) on the $6 \mathrm{D}$ supermanifold which furnishes the results of the horizontality condition, too. We would like to lay emphasis on the fact that the condition $\bar{\Psi}(x, \theta, \bar{\theta}) \Psi(x, \theta, \bar{\theta})=\bar{\psi}(x) \psi(x)$ is superior to the condition $\bar{\Psi}(x, \theta, \bar{\theta}) \gamma_{\mu} \Psi(x, \theta, \bar{\theta})=\bar{\psi}(x) \gamma_{\mu} \psi(x)$ because the former condition is without the Dirac gamma-matrices whereas the latter condition is endowed with it. The reason behind the superiority of the former over the latter is the fact that, so far, we have not been able to provide a nontrivial six-dimensional representation of the Dirac gammamatrices that are present on the l.h.s. of the latter restriction. It is obvious that the l.h.s. (of the latter restriction) is defined on the 6D supermanifold.

The insertions of the values of the secondary fields in terms of the basic fields of the Lagrangian density (2.1), into the super expansion (3.1), finally, lead to the following expansion of the superfields in terms of the nilpotent $\left(s_{(a) b}^{2}=0\right)$ and anticommuting $\left(s_{b} s_{a b}+\right.$ $\left.s_{a b} s_{b}=0\right)\left(\right.$ anti-)BRST transformations $s_{(a) b}$ of $(2.2)$ :

$$
\begin{aligned}
& \mathcal{B}_{\mu}^{(g)}(x, \theta, \bar{\theta})=A_{\mu}(x)+\theta\left(s_{a b} A_{\mu}(x)\right)+\bar{\theta}\left(s_{b} A_{\mu}(x)\right)+\theta \bar{\theta}\left(s_{b} s_{a b} A_{\mu}(x)\right) \\
& \mathcal{F}^{(c)}(x, \theta, \bar{\theta})=C(x)+\theta\left(s_{a b} C(x)\right)+\bar{\theta}\left(s_{b} C(x)\right)+\theta \bar{\theta}\left(s_{b} s_{a b} C(x)\right) \\
& \overline{\mathcal{F}}^{(a c)}(x, \theta, \bar{\theta})=\bar{C}(x)+\theta\left(s_{a b} \bar{C}(x)\right)+\bar{\theta}\left(s_{b} \bar{C}(x)\right)+\theta \bar{\theta}\left(s_{b} s_{a b} \bar{C}(x)\right) \\
& \Psi^{(g)}(x, \theta, \bar{\theta})=\psi(x)+\theta\left(s_{a b} \psi(x)\right)+\bar{\theta}\left(s_{b} \psi(x)\right)+\theta \bar{\theta}\left(s_{b} s_{a b} \psi(x)\right) \\
& \bar{\Psi}^{(g)}(x, \theta, \bar{\theta})=\bar{\psi}(x)+\theta\left(s_{a b} \bar{\psi}(x)\right)+\bar{\theta}\left(s_{b} \bar{\psi}(x)\right)+\theta \bar{\theta}\left(s_{b} s_{a b} \bar{\psi}(x)\right) .
\end{aligned}
$$

The above expressions provide the geometrical interpretations for (i) the transformations $s_{(a) b}$ (and corresponding generators $\left.Q_{(a) b}\right)$ as the translational generators along the Grassmannian directions of the 6D supermanifold, (ii) the nilpotency of $s_{(a) b}$ and $Q_{(a) b}$ as a couple of successive translations along $\theta$ and $\bar{\theta}$ directions of 6D supermanifold, and (iii) the anticommutativity properties of $s_{(a) b}$ and $Q_{(a) b}$ as encoded in the similar type of relations between translational generators along $\theta$ and $\bar{\theta}$ directions.

All the above key properties associated with the (anti-)BRST transformations for all the basic fields of QED (with Dirac fields), are encapsulated in the following

$$
\begin{aligned}
& s_{b} \Leftrightarrow Q_{b} \Leftrightarrow \operatorname{Lim}_{\theta \rightarrow 0} \frac{\partial}{\partial \bar{\theta}} \quad s_{a b} \Leftrightarrow Q_{a b} \Leftrightarrow \operatorname{Lim}_{\bar{\theta} \rightarrow 0} \frac{\partial}{\partial \theta} \\
& s_{(a) b}^{2}=0 \Leftrightarrow Q_{(a) b}^{2}=0 \Leftrightarrow\left(\frac{\partial}{\partial \theta}\right)^{2}=0\left(\frac{\partial}{\partial \bar{\theta}}\right)^{2}=0 \\
& s_{b} s_{a b}+s_{a b} s_{b}=0 \Leftrightarrow Q_{b} Q_{a b}+Q_{a b} Q_{b}=0 \Leftrightarrow \frac{\partial}{\partial \bar{\theta}} \frac{\partial}{\partial \theta}+\frac{\partial}{\partial \theta} \frac{\partial}{\partial \bar{\theta}}=0 .
\end{aligned}
$$

Thus, all the salient mathematical features of the BRST symmetries (as well as their generators) have been expressed in terms of the geometrical objects on the 6D supermanifold. 
Furthermore, the derivations of all the nilpotent (anti-)BRST symmetry transformations for QED (with Dirac fields) have been obtained together within the framework of the augmented superfield formalism in one stroke (cf. (4.1)) and their geometrical origin and interpretations have been provided.

\section{Conclusions}

One of the central results of our present investigation is the derivation of the nilpotent and anticommuting $\left(s_{b} s_{a b}+s_{a b} s_{b}=0\right.$ ) (anti-)BRST symmetry transformations $s_{(a) b}$ for the matter (Dirac) fields, the $U(1)$ gauge field and the (anti-)ghost fields together from a single restriction (cf. (4.1)) imposed on the six (4,2)-dimensional supermanifold (where all the superfields of the theory are defined). This is a completely new result because, in our earlier works [10-14], the above nilpotent symmetry transformations have been derived in two steps by exploiting (i) the horizontality condition, and (ii) its consistent extensions [1014], on the 6D supermanifold. It will be noted, however, that there is an interplay between the above two restrictions and they are not completely separate and independent. Thus, for a given $U(1)$ Abelian interacting 4D gauge theory, our present investigation provides a simpler derivation of the nilpotent (anti-)BRST transformations for all the fields of the theory within the framework of the superfield approach to BRST formalism.

The new restriction (4.1) on the 6D supermanifold is a gauge invariant restriction which leads to the derivation of the nilpotent (anti-)BRST symmetry transformations for all the fields (including the matter fields) of QED. Its gauge covariant version on the 6D supermanifold does not lead to the derivation of nilpotent (anti-)BRST symmetry transformations for the matter (Dirac) fields. It will be noted that the horizontality condition, on the other hand, is basically a gauge covariant restriction on the $6 \mathrm{D}$ supermanifold. In fact, the covariant version of (4.1) leads to the derivation of the exact nilpotent (anti-)BRST symmetry transformations for the gauge and (anti-)ghost fields only which are also the main results of the restriction due to the horizontality condition on the $6 \mathrm{D}$ supermanifold. Thus, the covariant version of the restriction (4.1) is equivalent, in some sense, to the restriction due to the horizontality condition. It is worth emphasizing that the horizontality condition $\tilde{F}^{(2)}=F^{(2)}$, reduces to the gauge invariant restriction on the $6 \mathrm{D}$ supermanifold only for the interacting $U(1)$ gauge theory (i.e. QED). This observation is, however, not true for the general case of the interacting 1-form non-Abelian gauge theories.

The importance of the gauge (i.e. BRST) invariant restriction in (4.1) comes out in its full blaze of glory in the context of superfield approach to BRST symmetries for the 1-form interacting non-Abelian 4D gauge theory where there is a coupling between the 1-form non-Abelian gauge field and the Dirac fields [18]. In fact, it has been shown in this very recent work [18], that the off-shell nilpotent symmetries for all the fields (of the (anti-)BRST invariant Lagrangian density of a given 4D 1-form interacting non-Abelian gauge theory) can be precisely computed due to the gauge invariant restriction (4.1) on the 
$6 \mathrm{D}$ supermanifold. In our present endeavour, there is a great deal of simplification in the derivation of the nilpotent symmetries for all the fields of the given 4D 1-form interacting $U(1)$ gauge theory (i.e. QED). This happens because of its Abelian nature. The situation is completely different in the case of the superfield approach to the derivation of the nilpotent symmetry transformations for the 1 -form interacting $4 \mathrm{D}$ non-Abelian theory where the non-Abelian nature of the theory generates interesting complications (see, e.g. [18]).

The horizontality condition of the usual superfield approach to BRST formalism has to be generalized so that one could obtain all the nilpotent symmetry transformations for all the fields of a given 4D p-form (non-)Abelian interacting gauge theory. This is due to the fact that the results, derived from the application of the horizontality condition on the 6D supermanifold alone, are partial in the sense that one obtains only the nilpotent symmetry transformations for the $p$-form gauge fields and the corresponding (anti)commuting (anti)ghost fields of the theory. The matter fields of the interacting $p$-form gauge theories remain untouched within the framework of the usual superfield formalism (with the theoretical arsenal of the horizontality condition alone). Thus, our present attempt is a step forward in the direction of the consistent and precise generalization of the horizontality condition where (i) the nilpotent symmetry transformations for all the fields (including the matter fields) of an interacting gauge theory are obtained, and (ii) the geometrical interpretations for all the properties associated with the BRST symmetries (and their generators) remain exactly the same as in the case of the application of the horizontality condition alone.

As a side remark, it is worthwhile to mention that the present off-shell nilpotent $\left(s_{(a) b}^{2}=\right.$ 0) (anti-)BRST symmetry transformations $s_{(a) b}$ for the interacting 1-form Abelian $U(1)$ gauge theory is derived for the specific choice of the gauge-fixing term (i.e. $-\frac{1}{2}(\partial \cdot A)^{2} \equiv$ $\left.B(\partial \cdot A)+\frac{1}{2} B^{2}\right)$ in the Feynman gauge. In this gauge, the ghost fields decouple from the rest of the physical fields of the theory so that any arbitrary state in the quantum Hilbert space is a direct product of the physical state and the ghost states. The subsidiary condition $Q_{b} \mid$ phys $>=0$, with the conserved and nilpotent BRST charge $Q_{b}$ on the physical state (first proposed by Curci and Ferrari [19,20]), plays a pivotal role in the proof of unitarity of the S-matrix of the theory by exploiting the so-called "quartet mechanism" (see, e.g., [21] for details). In general, for the non-Abelian gauge theory, the gauge-fixing term can include the ghost fields and, as a consequence, there would be an explicit coupling between the non-Abelian gauge fields and the (anti-)ghost fields. In this specific case, for the massless as well as massive gauge fields, a thorough discussion, devoted to the proof of unitarity of the S-matrix, has been carried out in $[20,22,23]$. However, for our present simple case of 1-form Abelian gauge theory in the Feynman gauge, the physicality criteria $\left(Q_{b} \mid\right.$ phys $\left.>=0\right)$, the nilpotency property $\left(Q_{b}^{2}=0\right)$ and the conservation of the BRST charge $\left(\dot{Q}_{b}=0\right)$ are good enough to shed some useful light on the unitarity of the theory (see, e.g., [21]).

It is interesting to check the validity the idea put forward in our present investigation, in different contexts (for totally different kinds of interacting systems). This will enable us to put our prescription on firmer footings as the gauge invariant restriction in (4.1) 
is a general restriction (valid for the (non-)Abelian gauge theories). In the superfield approach to BRST formalism, this prescription might be tested for the cases of (i) the complex scalar fields in interaction with the $U(1)$ gauge field, (ii) the gravitational theories which are very similar, in some sense, to the non-Abelian gauge theories (see, e.g., [16] for details on analogy), and (iii) the 2-form (non-)Abelian gauge fields and their interactions. Furthermore, it will be a challenging endeavour to obtain the results of the horizontality condition and its generalization $[12,13]$ (that lead to mathematically unique derivations of the nilpotent symmetry transformations for the matter fields) from a single restriction on the $6 \mathrm{D}$ supermanifold. The above pointed issues are some of the promising problems that are presently under investigation and our results will be reported elsewhere [24].

\section{References}

[1] Thierry-Mieg J 1980 J. Math. Phys. 212834 Thierry-Mieg J 1980 Nuovo Cimento A 56396

[2] Quiros M, De Urries F J, Hoyos J, Mazon M L and Rodrigues E 1981 J. Math. Phys. 22767

[3] Delbourgo R and Jarvis P D 1981 J. Phys. A: Math. Gen. 15611 Delbourgo R, Jarvis P D and Thompson G 1982 Phys. Lett. B 10925

[4] Bonora L and Tonin M 1981 Phys. Lett. B 9848 Bonora L, Pasti P and Tonin M 1981 Nuovo Cimento A 63353

[5] Baulieu L and Thierry-Mieg J 1982 Nucl. Phys. B 197477 Baulieu L and Thierry-Mieg J 1982 Nucl. Phys. B 228259 Alvarez-Gaumé L and Baulieu L 1983 Nucl. Phys. B 212255

[6] Hwang D S and Lee C -Y 1997 J. Math. Phys. 3830

[7] Dirac P A M 1964 Lectures on quantum mechanics, Belfer graduate school of science (Yeshiva University Press: New York)

[8] For a review, see, e.g., Sundermeyer K 1982 Constrained dynamics: Lecture notes in physics Vol 169 (Springer-Verlag: Berlin)

[9] Nakanishi N and Ojima I 1990 Covariant operator formalism of gauge theories and quantum gravity (World Scientific: Singapore)

[10] Malik R P 2004 Phys. Lett. B 584210 (Preprint hep-th/0311001) Malik R P 2004 J. Phys. A: Math. Gen. 375261 (Preprint hep-th/0311193)

Malik R P 2004 Int. J. Geom. Methods Mod. Phys. 1467 (Preprint hep-th/0403230) Malik R P 2005 Int. J. Mod. Phys. A 204899 (Preprint hep-th/0402005) Malik R P 2005 Int. J. Mod. Phys. A 207285 (Erratum) (Preprint hep-th/0402005)

[11] Malik R P 2005 Mod. Phys. Lett. A 201767 (Preprint hep-th/0402123) Malik R P 2006 Eur. Phys. J. C 45513 (Preprint hep-th/0506109)

[12] Malik R P 2006 Eur. Phys. J. C 47227 (Preprint hep-th/0507127) Malik R P 2005 Augmented superfield spproach to unique nilpotent symmetries for complex scalar fields in QED (Preprint hep-th/0508226) (2006 Eur. Phys. J. C 49 at press) 
[13] Malik R P and Mandal B P 2006 Eur. Phys. J. C 47219 (Preprint hep-th/0512334)

[14] Malik R P 2005 Superfield approach to exact and unique nilpotent symmetries, (Preprint hep-th/0510029)(To appear in the Proc. of the conference on "Supersymmetries and Quantum Symmetries" (SQS:'05) held at Bogoliubov Laboratory of Theoretical Physics, JINR, Dubna, from 27 July till 31st July 2005).

[15] Henneaux M and Teitelboim C 1992 Quantization of gauge systems (Princeton University Press: Princeton, NJ)

[16] Weinberg S 1996 The quantum theory of fields: modern applications vol 2 (Cambridge University Press: Cambridge)

[17] Aitchison I J R and Hey A J G 1982 Gauge theories in particle physics: a practical introduction (Adam Hilger: Bristol)

[18] Malik R P 2006 An alternative to horizontality condition in superfield spproach to BRST symmetries (Preprint hep-th/0603049)

[19] Curci G and Ferrari R 1975 Nuovo Cimento A 30155

[20] Curci G and Ferrari R 1976 Nuovo Cimento A 35273

[21] Kogo T and Ojima I 1979 Prog. Theo. Phys. (Suppl) 661

[22] Curci G and Ferrari R 1976 Phys. Lett. B 6391

[23] Curci G and Ferrari R 1976 Nuovo Cimento A 351 Curci G and Ferrari R 1978 Nuovo Cimento A 47555 (Erratum)

[24] Malik R P, in preparation 